\title{
GROUP BIALGEBRAS AND PERMUTATION BIALGEBRAS
}

\author{
MARTIN CROSSLEY \\ Department of Mathematics, Swansea University, Swansea SA2 8PP, Wales \\ e-mail:M.D.Crossley@Swansea.ac.uk
}

(Received 17 May 2012; revised 17 September 2012; accepted 1 November 2012; first published online 25 February 2013)

\begin{abstract}
Malvenuto and Reutenauer (C. Malvenuto and C. Reutenauer, Duality between quasi-symmetric functions and the Solomon descent algebra, J. Algebra 177 (1995), 967-982) showed how the total symmetric group ring $\oplus_{n} \mathbf{Z} \Sigma_{n}$ could be made into a Hopf algebra with a very nice structure which admitted the Solomon descent algebra as a sub-Hopf algebra. To do this they replaced the group multiplication by a convolution product, thus distancing their structure from the group structure of $\Sigma_{n}$. In this paper we examine what is possible if we keep to the group multiplication, and we also consider the question for more general families of groups. We show that a Hopf algebra structure is not possible, but cocommutative and non-cocommutative counital bialgebras can be obtained, arising from certain diagrams of group homomorphisms. In the case of the symmetric groups we note that all such structures are weak in the sense that the dual algebras have many zero-divisors, but structures which respect descent sums can be found.
\end{abstract}

2010 Mathematics Subject Classification. 16T05, 20B30, 16S34, 20 C05.

1. Group bialgebras. Malvenuto and Reutenauer in [2] showed how a Hopf algebra structure can be defined on the total symmetric group ring $\oplus_{n} \mathbf{Z} \Sigma_{n}$ in such a way that the Solomon descent algebra is a sub-Hopf algebra. In that work, and related papers such as [1], the product used in the Hopf algebra structure was the convolution product, not the composition product arising directly from the group operation in $\Sigma_{n}$. In this paper we investigate how much can be done if we use the composition product so that the multiplicative structure is as faithful as possible to the group structure of $\Sigma_{n}$. We start by considering bialgebra structures on arbitrary families of finite groups before specializing to the symmetric groups.

Let $G_{1}, G_{2}, \ldots$ be any family of finite groups. Let $G_{0}$ be the trivial group and define

$$
\mathbf{Z} G=\bigoplus_{n} \mathbf{Z} G_{n},
$$

with the grading, where $G_{n}$ has degree $n$. The product is given by defining $g . h$ to be the usual product in $G_{n}$ if $|g|=|h|=n$, and 0 if $|g| \neq|h|$. This product is associative, non-commutative (unless every $G_{n}$ is abelian) and non-unital. This latter fact means there can be no antipode and, hence no Hopf algebra structure on $\mathbf{Z} G$ based on this product. We can, however, make $\mathbf{Z} G$ a bialgebra. Of course, one way would be to make each group element group-like; this gives the direct sum of the usual Hopf algebra structures on each group ring $\mathbf{Z} G_{n}$, and forms a bialgebra which is coassociative but not counital. We will take a different approach by constructing counital, coassociative 
bialgebras in which the coproduct $\Delta$ preserves the grading, i.e. $\Delta$ restricts, for each $n$, to a function

$$
\Delta_{n}: \mathbf{Z} G_{n} \longrightarrow \bigoplus_{i=0}^{n}\left(\mathbf{Z} G_{i} \otimes \mathbf{Z} G_{n-i}\right)
$$

This means that we can write

$$
\Delta_{n}=\sum_{i=0}^{n} \Delta_{n, i}, \text { where } \Delta_{n, i}: \mathbf{Z} G_{n} \rightarrow \mathbf{Z} G_{i} \otimes \mathbf{Z} G_{n-i} \cong \mathbf{Z}\left(G_{i} \times G_{n-i}\right) .
$$

Multiplicativity of $\Delta$ is equivalent to each $\Delta_{n, i}$ being a ring homomorphism. An obvious class of ring homomorphisms $\mathbf{Z} G_{n} \rightarrow \mathbf{Z}\left(G_{i} \times G_{n-i}\right)$ consists of those induced by a group homomorphism $G_{n} \rightarrow G_{i} \times G_{n-i}$, i.e. a pair of group homomorphisms $G_{n} \rightarrow G_{i}, G_{n} \rightarrow G_{n-i}$. Although not all ring homomorphisms arise in this way, it is a rich enough class to be interesting and makes it easier to analyse the properties of $\Delta$. In particular, we have the following result.

THEOREM 1. Let $\Delta: \mathbf{Z} G \rightarrow \mathbf{Z} G \otimes \mathbf{Z} G$ be a graded ring homomorphism such that each $\Delta_{n}: \mathbf{Z} G_{n} \longrightarrow \bigoplus_{i=0}^{n}\left(\mathbf{Z} G_{i} \otimes \mathbf{Z} G_{n-i}\right)$ is induced from a pair of group homomorphisms $\Delta_{n, i}^{L}: G_{n} \rightarrow G_{i}$ and $\Delta_{n, i}^{R}: G_{n} \rightarrow G_{n-i}$. Then

(1) $\Delta$ is counital if and only if $\Delta_{n, n}^{L}=\Delta_{n, 0}^{R}=1_{G_{n}}$ for each $n$.

(2) $\Delta$ is coassociative if and only if for all $0 \leq i \leq j \leq n$ we have $\Delta_{j, i}^{L} \circ \Delta_{n, j}^{L}=\Delta_{n, i}^{L}$, $\Delta_{n-i, j-i}^{R} \circ \Delta_{n, i}^{R}=\Delta_{n, j}^{R}$ and $\Delta_{j, i}^{R} \circ \Delta_{n, j}^{L}=\Delta_{n-i, j-i}^{L} \circ \Delta_{n, i}^{R}$.

(3) $\Delta$ is cocommutative if and only if for all $0 \leq i \leq n, \Delta_{n, i}^{L}=\Delta_{n, n-i}^{R}$.

Proof. We prove (2), the proofs of (1) and (3) being similar. For an element $g \in G_{n}, \Delta(g)$ is equal to the sum (over $0 \leq j \leq n$ ) of the terms $\left(\Delta_{n, j}^{L}(g), \Delta_{n, j}^{R}(g)\right) \in$ $\mathbf{Z}\left(G_{j} \times G_{n-j}\right)$. Hence, $(\Delta \otimes 1) \circ \Delta(g)$ is the sum (over $\left.0 \leq i \leq j \leq n\right)$ of the terms $\left(\left(\Delta_{j, i}^{L} \circ \Delta_{n, j}^{L}\right)(g),\left(\Delta_{j, i}^{R} \circ \Delta_{n, j}^{L}\right)(g), \Delta_{n, j}^{R}(g)\right)$ in $\mathbf{Z}\left(G_{i} \times G_{j-i} \times G_{n-j}\right)$. Writing out the corresponding expression for $(1 \otimes \Delta) \circ \Delta(g)$ leads to the stated result.

Hence, a coassociative, counital coproduct $\Delta$ is built from commuting pairs of group homomorphisms $\Delta_{n, n-1}^{L}: G_{n} \rightarrow G_{n-1}, \Delta_{n, 1}^{R}: G_{n} \rightarrow G_{n-1}$. In conclusion, we have the following.

THEOREM 2. For any family of group homomorphisms $\Delta_{n, n-1}^{L}: G_{n} \rightarrow G_{n-1}, \Delta_{n, 1}^{R}$ : $G_{n} \rightarrow G_{n-1}$ that commute in the sense that

$$
\Delta_{n, n-1}^{L} \circ \Delta_{n+1,1}^{R}=\Delta_{n, 1}^{R} \circ \Delta_{n+1, n}^{L},
$$

there exists a degree-preserving, counital, coassociative coproduct $\Delta$ on $\bigoplus_{n} \mathbf{Z} G_{n}$ given by

$$
\Delta(g)=\sum_{i=0}^{n}\left(\Delta_{i+1, i}^{L} \circ \cdots \circ \Delta_{n, n-1}^{L}\right)(g) \otimes\left(\Delta_{n-i+1,1}^{R} \circ \cdots \circ \Delta_{n, 1}^{R}\right)(g),
$$

where $n=|g|$. With this coproduct and the composition product $\bigoplus_{n} \mathbf{Z} G_{n}$ is a bialgebra. 
In other words, such a bialgebra arises whenever we have a commutative diagram

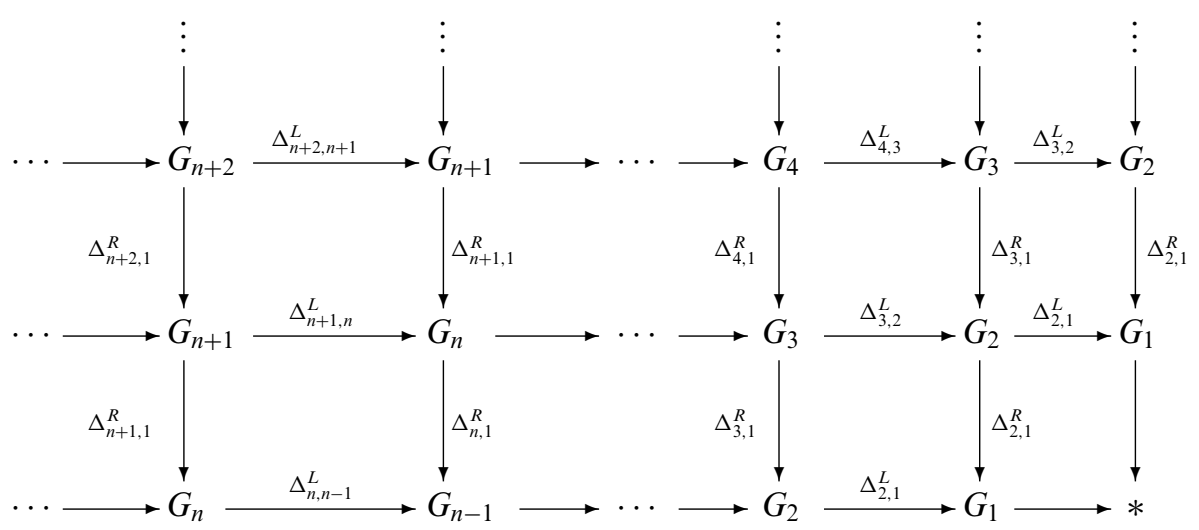

in which each horizontal arrow is some $\Delta_{k, k-1}^{L}$ for appropriate choice of $k$, and each vertical arrow is some $\Delta_{k, 1}^{R}$. In particular, each row is equal to a rightward shift of the row beneath it, and each column is equal to a downward shift of the column to the right of it.

If the coproduct is cocommutative, then condition (1) is automatic. Hence, we have the following.

THEOREM 3. Any family of group homomorphisms $\Delta_{n, 1}^{R}: G_{n} \rightarrow G_{n-1}$ gives rise to a degree-preserving, counital, coassociative, cocommutative coproduct on $\bigoplus_{n} \mathbf{Z} G_{n}$ given by

$$
\Delta(g)=\sum_{i=0}^{n}\left(\Delta_{i+1,1}^{R} \circ \cdots \circ \Delta_{n, 1}^{R}\right)(g) \otimes\left(\Delta_{n-i+1,1}^{R} \circ \cdots \circ \Delta_{n, 1}^{R}\right)(g),
$$

where $n=|g|$, that makes $\bigoplus_{n} \mathbf{Z} G_{n}$ with the composition product into a bialgebra.

This corresponds to the case where, in the above diagram, the vertical and horizontal arrows coincide, so the diagram collapses to a chain

$$
\cdots \longrightarrow G_{n} \longrightarrow G_{n-1} \longrightarrow \cdots \longrightarrow G_{1} \longrightarrow * .
$$

Hence, Theorem 3 tells us that to any such sequence of group homomorphisms, there is associated a cocommutative bialgebra on $\bigoplus \mathbf{Z} G_{n}$.

For example, if we take each $G_{n}$ to be the trivial group (with, necessarily, each homomorphism being trivial) then the resulting bialgebra is the dual of the polynomial algebra $\mathbf{Z}[x]$ in which $x$ is group-like.

For a less trivial example, we may take each $G_{n}$ to be the cyclic group of order 2 , and each homomorphism to be the identity. In that case the bialgebra is the dual of $\mathbf{Z}[x, y] /(x y)$ with $x$ and $y$ group-like.

For a general family of groups, we can always find a cocommutative coproduct on $\bigoplus \mathbf{Z} G_{n}$ by taking each homomorphism $G_{n} \rightarrow G_{n-1}$ to be trivial. Moreover, in all but the most trivial cases, we may also construct non-cocommutative coproducts, for example by taking all the $\Delta_{n, n-1}^{L}$ to be trivial, and (at least one of) the $\Delta_{n, 1}^{R}$ to be non-trivial. 
2. Permutation bialgebras. When we specialize to the family of symmetric groups, $G_{n}=\Sigma_{n}$ for each $n$, we immediately hit restrictions. The simplicity of the alternating group, $A_{n}$, for $n \neq 4$ implies that each homomorphism $\Delta_{n, n-1}^{L}, \Delta_{n, 1}^{R}$ has an image of order at most 2. Such a small image implies the existence of many zero-divisors in the dual algebra. For, suppose that $x$ is a dual element which, for simplicity, we will assume to be homogeneous of degree $n$, that is zero on the image of $\Delta_{n+1, n}^{L}$. In other words, $x$ is a linear function $Z \Sigma_{n} \rightarrow \mathbf{Z}$ such that $x \circ \Delta_{n+1, n}^{L}=0$. Then in the dual algebra $\oplus_{n} \operatorname{Hom}\left(\mathbf{Z} \Sigma_{n}, \mathbf{Z}\right)$, any product $x . y$ will be 0 if $y$ has positive degree. This is because if $y$ is a homogeneous element of degree $m$, then $x . y$ is the linear function given by $g \mapsto(x \circ$ $\left.\Delta_{m+n, n}^{L}\right)(g) \cdot\left(y \circ \Delta_{m+n, n}^{R}\right)(g)$ for all $g \in \Sigma_{m+n}$. Since $\Delta_{m+n, n}^{L}=\Delta_{n+1, n}^{L} \circ \cdots \circ \Delta_{m+n, m+n-1}^{L}$, it follows that $\left(x \circ \Delta_{m+n, n}^{L}\right)(g)=0$ for all $g$ and, hence, $x \cdot y=0$. Similarly, if $x \circ \Delta_{n, 1}^{R}=0$ then all products $y . x$ will be zero if $|y|>0$. Since the image of each of these maps has at most two elements, there will be many dual elements that are zero on either one or both images, and thus many zero-divisors.

Nevertheless, the desired structure is possible.

THEOREM 4. There are graded, counital, coassociative, cocommutative coproducts on $\oplus \mathbf{Z} \Sigma_{n}$ which make this into a bialgebra.

There are graded, counital, coassociative, non-cocommutative coproducts on $\oplus \mathbf{Z} \Sigma_{n}$ which make this into a bialgebra.

Proof. One cocommutative coproduct is given by defining both $\Delta_{n, n-1}^{L}$ and $\Delta_{n, 1}^{R}$ to be the homomorphism that maps all even permutations to the identity and all odd ones to a given transposition in $\Sigma_{n-1}$. Clearly, there are many other choices for $\Delta_{n, n-1}^{L}$, and the choice in defining $\Delta_{n, i}$ in terms of $\Delta_{n, i}^{L}$ and $\Delta_{n, i}^{R}$ gives rise to further possibilities.

One non-cocommutative coproduct is given by defining $\Delta_{n, n-1}^{L}$ as above and setting $\Delta_{n, 1}^{R}$ to be the trivial homomorphism for all $n$. Again, there are clearly many other choices.

Following Malvenuto-Reutenauer [2] we would also like a coproduct which respects the Solomon descent algebra [4]. This is also possible.

THEOREM 5. There are graded, counital, coassociative, cocommutative coproducts on $\oplus \mathbf{Z} \Sigma_{n}$ which respect descent sums, making this into a bialgebra which admits the Solomon descent algebra as a sub-bialgebra.

There are graded, counital, coassociative, non-cocommutative coproducts on $\oplus \mathbf{Z} \Sigma_{n}$ which respect descent sums, making this into a bialgebra which admits the Solomon descent algebra as a sub-bialgebra.

To clarify these statements, we are not making any claims about any pre-existing coproduct on the Solomon descent algebra, since none of the coproducts hitherto described in the literature for the Solomon descent algebra are compatible with our product (that is to say, with the original product on that algebra) to give a bialgebra structure. We are merely claiming that with the original algebra structure it is possible to find a coproduct on $\oplus \mathbf{Z} \Sigma_{n}$ that preserves the sub-algebra spanned by descent sums and thus gives a bialgebra structure on the Solomon descent algebra that is a subbialgebra of $\oplus \mathbf{Z} \Sigma_{n}$. We also draw the reader's attention to the fact that the issues highlighted in [3], about the difficulties in putting a bialgebra structure on a sub-object of a bialgebra, are circumvented by the very explicit nature of our constructions. 
Proof. To respect descent sums, each homomorphism $\Delta_{n, n-1}^{L}, \Delta_{n, 1}^{R}$ must respect descent sums. For $n \neq 4$, this requires that the image of each homomorphism $\Delta_{n, n-1}^{L}$, $\Delta_{n, 1}^{R}$ consists of group elements which are themselves descent sums. The only such elements in $\mathbf{Z} \Sigma_{n}$ are the identity and the permutation $d_{n}=(n, n-1, \ldots, 1)$. Thus, for a cocommutative coproduct we may define $\Delta_{n, n-1}^{L}=\Delta_{n, 1}^{R}$ to be the homomorphism that takes even permutations to the identity and odd permutations to $d_{n-1}$. Further choices arise since for any given $n$ we may set $\Delta_{n, n-1}^{L}=\Delta_{n, 1}^{R}$ to be the trivial homomorphism, and again we have choices about defining each $\Delta_{n, i}$.

For $n=4$ there is one further possibility: We may define $\Delta_{4,3}^{L}=\Delta_{4,1}^{R}$ to be the epimorphism $\mathbf{Z} \Sigma_{4} \rightarrow \mathbf{Z} \Sigma_{3}$ given by

$$
2134 \mapsto 213, \quad 1324 \mapsto 132, \quad 1243 \mapsto 213
$$

since this also respects descent sums.

For a non-cocommutative coproduct we may proceed as before: taking any of these cocommutative coproducts and replacing all the $\Delta_{n, 1}^{R}$ by the trivial homomorphism, or by similarly trivializing all of the $\Delta_{n, n-1}^{L}$.

If $\Delta_{4,3}^{L}=\Delta_{4,1}^{R}$ are epimorphisms, then more of the products in the dual algebra will be non-trivial. However, even in this case the descent-preservation forces a high level of triviality. Since $d_{n} \in A_{n}$ if $n \equiv 0$ or $1 \bmod 4$, it follows that any four-fold composite $\Delta_{n, n-4}^{L}$ or $\Delta_{n, 4}^{R}$ will be trivial. Hence, in the dual algebra, any product $x . y$ where $|x| \geq 4$ will be zero unless $y$ is the unit, or $x$ is the identity element in $\Sigma_{|x|}$.

\section{REFERENCES}

1. M. Aguiar, W. Ferrer and W. Moreira, The smash product of symmetric functions. Extended abstract. Preprint (2004). Available at http://arxiv.org/pdf/math.CO/0412016.pdf. Accessed on August 23rd, 2010.

2. C. Malvenuto and C. Reutenauer, Duality between quasi-symmetric functions and the Solomon descent algebra, J. Algebra 177 (1995), 967-982.

3. W. Nichols and M. Sweedler, Hopf algebras and combinatorics, Contemp. Math. 6 (1982), 49-84.

4. L. Solomon, A Mackey formula in the group ring of a Coxeter group, J. Algebra 41 (1976), 255-268. 